\title{
Optical force on diseased blood cells: towards the optical sorting of biological matter
}

\author{
Juan Sebastian Totero Gongora ${ }^{1}$ and Andrea Fratalocchi ${ }^{1, *}$ \\ 1PRIMALIGHT, Faculty of Electrical Engineering, Applied Mathematics and Computational Science, \\ King Abdullah University of Science and Technology (KAUST), Thuwal 23955-6900, Saudi Arabia \\ ${ }^{*}$ Corresponding author. Email: andrea.fratalocchi@kaust.edu.sa - Tel: +966(12)8080348
}

\begin{abstract}
By employing a series of massively parallel $a b$-initio simulations, we study how optical forces act on biological matter subject to morphological disease. As a representative case study, we here consider the case of Plasmodium Falciparum on red blood cells (RBC) illuminated by a monochromatic plane wave. Realistic parameters for the geometry and the refractive index are then taken from published experiments. In our theoretical campaign, we study the dependence of the optical force on the disease stage for different incident wavelengths. We show that optical forces change significantly with the disease, with amplitude variation in the hundreds of $\mathrm{pN}$ range. Our results open up new avenues for the design of new optical systems for the treatment of human disease.
\end{abstract}




\section{Introduction}

In recent years, the interest in the study of the interaction between light and biological matter has largely increased. While optics has historically been mainly used for imaging applications, light has now become a tool of manipulation and direct interaction with biological samples. It is well known that when light impinges on an object it exerts a small force on it, whose values are in the range of tens of $\mathrm{pN}$ for micro sized specimens. Such optical force has two fundamental components: the gradient force, which is related to the electrostatic interaction between the polarization charges in the object and the field distribution itself, and the scattering force, due to the photon momentum transfer $[1,2]$. The scattering part of the optical force depends on the wavenumber distribution of both the incident and scattered fields. Application examples of optical forces are found in optical tweezers, where a focused light beam is exploited to trap and transport micro/nano particles [3-6]. In biology, optical tweezers are usually employed as a non-invasive technique to manipulate cells and their sub-cellular components $[7,8]$. While optical tweezers usually employ gradient forces, scattering forces have been recently investigated and a series of counterintuitive dynamics has been demonstrated, including fully optical tractor beams [9-11]. These techniques, including more pioneering idea such as e.g., cell optical sorting [12] and in-vivo manipulation [13] that are still under investigation, require a precise understanding on how the optical pressure affects biological organisms. This calls for the development of precise numerical methods, whose development will be crucial for applications of the aforementioned techniques. In this respect, ab-initio techniques represent a very important tool that can provide quantitative answers to the problem. Among the different approaches, the Finite Differences Time Domain (FDTD) method, yields a very flexible technique to study any experimental condition, as it is based on the numerical solution of Maxwell equations with no approximation [14]. Despite FDTD techniques have been applied in biology to simulate scattering from tissues and cells [15-17], nothing has been done in the direct computation of optical forces on bio samples.

In this work, we computed the optical forces acting on biological samples using FDTD simulations. In our FDTD approach, which explicitly considers the dispersive properties of the sample and of the environment, optical forces are expressed in terms of the Maxwell stress tensor formulation [18]. As an interesting case study, we measured the variation of optical force on a Red Blood cell when the morphology and the internal structure are changed, as in the case of RBC infected by Plasmodium Falciparum. Our results show that the optical force changes significantly with the morphology of the cell, allowing to sort RBC according to their disease progression.

This article is organized as follows. In section 1 we introduce the RBC model, describing its main properties as well as the typical deformation occurring in the different stages of a $P$. Falciparum infection. In section 2 we describe our FDTD calculation of optical forces within the Maxwell Stress Tensor formalism. In Section 3 we discuss the results of our simulations. Section 4 finally presents our conclusions. 


\section{Materials and Methods}

In order to simulate a realistic biological sample, we considered Red Blood Cells, whose internal structure allows for a detailed optical modeling. Numerical simulations have shown that the presence of the cellular membrane does not provide significant contribution to the scattering properties of the RBC [19]. Moreover, in a healthy RBC, the cytoplasm of the cell is mainly homogeneous and it is primarily composed of Hemoglobin (Hgb) [20]. In terms of cell morphology, a healthy RBC exhibits a characteristic biconcave shape [21]. However, in the presence of an infection, the morphology and the internal structure of the RBC can change significantly. In some specific cases, such changes can be helpful in the diagnosis of the disease, as in the case of blood-strip based diagnosis of malaria [22]. In the past, much effort has been carried to model the optical response of RBCs in order to extract morphological information from scattering experiments and microscope measurements [19, 23, 24]. Among the different models proposed, the Evans-Fung model is widely used to represent the biconcave shape of a healthy RBC under different environmental conditions [25]. In the Evans-Fung model, which assumes an axisymmetric RBC, the profile of the cell can be represented by the thickness $\mathrm{D}(\mathrm{x})$ along the major axis (See Fig. 1a, red solid line), whose expression reads:

$$
D(x)=\sqrt{1-\left(\frac{x}{R_{0}}\right)^{2}}\left[C_{0}-C_{2}\left(\frac{x}{R_{0}}\right)^{2}+C_{4}\left(\frac{x}{R_{0}}\right)^{4}\right]
$$

In Eq. (1) $\mathrm{R}_{0}$ is the radius of the cell along the major axis, $\mathrm{C}_{0}$ represents the central thickness and $\mathrm{C}_{2}, \mathrm{C}_{4}$ determine the biconcave shape curvature. These sets of parameters are usually extracted from scattering experiments or by directly fitting microscope or tomographic measurements of RBC samples $[20,26]$. Typical values for a healthy RBC (hRBC) are

$\mathrm{R}_{0}=3.91(\mu \mathrm{m}), \mathrm{C}_{0}=0.81(\mu \mathrm{m}), \mathrm{C}_{2}=7.38(\mu \mathrm{m}), \mathrm{C}_{4}=-4.39(\mu \mathrm{m})$

which correspond to an isotonic RBC [25].

\subsection{Optical modeling of Red Blood Cells infected by P. Falciparum}

As already mentioned, however, the presence of an infection can radically change the properties of the RBCs. In order to address a realistic case, we considered the modifications occurring in a red blood cell infected by Plasmodium Falciparum, one of the parasites responsible of malaria in humans. In the different stages of the parasite reproduction cycle, which is conventionally divided into ring ( $\mathrm{rRBC}$ ), trophozoite (tRBC) and schizont (sRBC) stages, the internal structure and the morphology of the infected RBC (iRBC) undergo radical changes (Fig. 1b). From a chemical point of view, the parasite consumes part of the Hgb from the RBC cytoplasm and produces high-refractive index Hemozoin crystals [27, 28]. From a morphological point of view, the iRBC loses its typical axisymmetric biconcave shape due to the growth of the parasite and, in the trophozoite and schizont stages, the cellular membrane of the iRBC becomes irregular due to the appearance of characteristic protuberances. It follows that a realistic optical description of the iRBC must include all the morphological changes at the different stages of infection, the presence of the parasite and the variation of concentration of Hgb in the cell cytoplasm. In our 
work, we modeled the irregular shape of an iRBC (Fig. 1a, blue dashed line) by applying a deformation displacement $\mathrm{d} \mathbf{r}$ with definite statistical properties (Fig. 1a, yellow arrows) to every point of the curve describing a healthy RBC (Fig. 1a, solid red line) [29]. The expression of the deformation factor, which is directed along the normal to the surface, reads:

$$
d \boldsymbol{r}(s)=G_{\boldsymbol{\mu}}(s) \cdot A(s) \cdot \boldsymbol{n}(s),
$$

where $s$ is the curvilinear coordinate along the surface, $\mathbf{n}(s)$ is the normal unit vector, $G_{\mu}(s)$ is a Gaussian correlated random variable with mean value $\mu$ and correlation length $l_{c}$ and $A(s)$ is an asymmetry multiplicative factor. The characteristic shape of the infected RBCs can be obtained by considering an asymmetry factor defined as:

$$
A(s)=\exp \left[-\frac{\left[\mathrm{l}(\mathrm{s})-\mathrm{L}_{0}\right]^{2}}{2\left(L_{2}\right)^{2}}\right]-\Delta L
$$

where $\mathrm{l}(\mathrm{s})$ represents the length of the curve as a function of the curvilinear coordinate $\mathrm{s}$ and $\mathrm{L}_{0}, \mathrm{~L}_{2}$ and $\Delta \mathrm{L}$ are length parameters which can be extracted from microscope or tomographic images of infected RBCs. As a result, Equations (2) and (3) provide an intuitive and physical model for the morphological changes occurring in an iRBC (Fig. 1b) and, due to the realizationdependent nature of the deformation factor, they allow for a statistical description of infected RBCs populations. In order to address the structural changes occurring in the interior of an iRBC, we modeled the parasite invading the iRBC as a rugged ellipsoid, whose geometrical parameters correspond to the experimental measurements reported in [30, 31]. As discussed in [32], the internal structure of the parasite has been divided into a cytoplasmic shell and a Hemozoin-rich core, with refractive index $\mathrm{n}_{\mathrm{PF}}(\lambda)$ and $\mathrm{n}_{\mathrm{H}}(\lambda)$, as functions of the wavelength, respectively [28]. The optical properties of $\mathrm{Hgb}$ as a function of its concentration have been subject of intensive study and several models have been proposed to describe the complex refractive index of a Hgb solution $\tilde{n}_{\mathrm{Hgb}}(\lambda)$ as a function of wavelength. Following [27], we computed the complex refractive index for Hgb concentrations of $309 \mathrm{~g} / \mathrm{l}$ (healthy cell) , 293g/l (rRBC) , 233g/l (tRBC) and $187 \mathrm{~g} / \mathrm{l}$ (sRBC) using the models and data included in [33-36].

\section{Theoretical analysis and FDTD implementation}

We compute optical forces with our massively parallel simulator NANOCPP, which is an homemade parallel c++ FDTD code that scales up to hundreds of thousands of processors [37]. In the FDTD algorithm, Maxwell's equations are solved simultaneously in space and time, on a lattice specifically designed to enforce divergence conditions and within a second order time accurate algorithm [14]. FDTD provides a full knowledge over all the field components, both in the time and space domain, with arbitrary precision. In order to reduce the computational time, we resorted to parallel computing techniques and we decompose the spatial domain across a large number of processors, which solve Maxwell's equations in parallel. In our implementation, all the exchange of information among the processors is based on the Message-PassageInterface (MPI) standard. Electromagnetic sources are then included using an exact implementation based on the Total-Field-Scattered-Field (TFSF) formulation, including fully customizable Uniaxial-Perfectly-Matched-Layer (UPML) to simulate realistic open systems [14]. 
We compute the optical force in terms of the Maxwell Stress Tensor $(\stackrel{\leftrightarrow}{\mathbf{T}})_{\mathrm{ij}}$, defined as:

$$
(\stackrel{\mathrm{T}}{\mathrm{T}})_{\mathrm{ij}} \stackrel{\text { def }}{=} \varepsilon_{0}\left(\mathrm{E}_{\mathrm{i}} \mathrm{E}_{\mathrm{j}}-\frac{1}{2} \delta_{\mathrm{ij}} \mathrm{E}^{2}\right)+\mu_{0}\left(\mathrm{H}_{\mathrm{i}} \mathrm{H}_{\mathrm{j}}-\frac{1}{2} \delta_{\mathrm{ij}} \mathrm{H}^{2}\right), \mathrm{i}=\mathrm{x}, \mathrm{y}, \mathrm{z}
$$

where $\varepsilon_{0}$ is the vacuum permittivity, $\mu_{0}$ is the vacuum permeability, $\delta_{\mathrm{ij}}$ is the Kronecker delta and $\mathbf{E}=\left(E_{x}, E_{y}, E_{z}\right)$ and $\mathbf{H}=\left(H_{x}, H_{y}, H_{z}\right)$ are the electric and magnetic fields , respectively [18]. Given Eq. (4), the optical force $\mathbf{F}=\left(\mathrm{F}_{\mathrm{x}}, \mathrm{F}_{\mathrm{y}}, \mathrm{F}_{\mathrm{z}}\right)$ exerted by an electromagnetic wave impinging on an object is then obtained from the time average of the flux of the Maxwell Stress Tensor over an arbitrary enclosing surface $S$, namely

$$
\begin{gathered}
\overrightarrow{\mathbf{F}}=\left\langle\oiint_{S} \stackrel{\leftrightarrow}{\mathbf{T}} \cdot \mathrm{d} \overrightarrow{\mathbf{S}}\right\rangle_{\mathrm{T}} \\
\mathrm{F}_{\mathrm{i}}=\left\langle\sum_{\mathrm{j}} \oiint_{S} \mathrm{~T}_{\mathrm{ij}} \mathrm{d} S_{\mathrm{j}}\right\rangle_{\mathrm{T}}, \mathrm{i}=\mathrm{x}, \mathrm{y}, \mathrm{z},
\end{gathered}
$$

Where the time average is defined over an optical cycle $\mathrm{T}$ as follows:

$$
\langle f(t)\rangle_{T}=\frac{1}{T} \int_{0}^{T} f(t)
$$

The field-dependent nature of $(\stackrel{\mathbf{T}}{\mathrm{T}})_{\mathrm{ij}}$ simplifies the computation of the force $\mathrm{F}_{\mathrm{i}}$ in a FDTD simulation, where the electric and magnetic fields are available in every point of the discretized domain. Equations (3)-(5) are computed by a trapezoidal integration of the field components along the surface $S$, and by time averaging the resulting values. With this implementation, optical forces acting on a generic system can be computed with an arbitrary precision, limited in principle only by the available computational resources.

\section{Results and Discussion}

Figure 2a summarizes the FDTD setup used in our numerical experiments: a two-dimensional RBC is placed inside a $9 \mu \mathrm{m} \times 4 \mu \mathrm{m}$ TFSF region, where a monochromatic plane wave of wavelength $\lambda$ propagates along the $\hat{\mathbf{z}}$ direction. The medium surrounding the RBC we consider dispersive plasma with refractive index $\mathrm{n}_{\mathrm{m}}(\lambda)$ [38]. The incident power density is fixed at $8.4 \mathrm{~mW} / \mu \mathrm{m}^{2}$, which matches the actual value used in in-vivo experiments with optical tweezers and therefore lies below the radiation damage threshold of RBCs [13]. A typical spatial distribution of the total electromagnetic energy is displayed in Fig. 2b, where a healthy RBC is illuminated with a $\lambda=480 \mathrm{~nm}$ plane wave. To eliminate any artificial boundary effect in the calculation of the optical force, we employed a thick (60 points) UPML layer. We considered a spatial domain corresponding to a volume of $10 \mu \mathrm{m} \times 5 \mu \mathrm{m}$, with a discretization of $2500 \times 1250$ points, equivalent to a resolution of $\mathrm{dx}=\mathrm{dz}=4 \mathrm{~nm}$. This corresponds to a resolution between 65 and 286 points per internal wavelength, which ensures a very high-accuracy in the force computation. In our simulations, we considered wavelengths in the both visible and IR regions, which can penetrate the tissues. The integration surface $S$ is symmetrically placed $100 \mathrm{~nm}$ apart 
from the RBC surface, sufficiently near to capture the near-field features of the scattered field. In our simulations, we considered realistic dispersion parameters of the Hemoglobin, as taken from the literature [33-36]. Figure 3 reports the behavior of the refractive index $\mathrm{n}_{\mathrm{HGB}}$ and the absorption $\mathrm{k}_{\mathrm{HGB}}$ for different wavelengths and for different concentrations. As seen from Fig. 3, both quantities $\mathrm{n}_{\mathrm{HGB}}$ and $\mathrm{k}_{\mathrm{HGB}}$ vary strongly as a function of the wavelength and the concentration.

In our FDTD experiments, we measured the component of the force in the propagation direction $\left(\mathrm{F}_{\mathrm{z}}\right)$. In order to investigate the dependence of the optical force to the geometrical parameters, we performed an extensive massively parallel campaign, composed of approx. 5M of CPU hours on 4096 processors. In each simulation, we change both the frequency and the morphology of the RBC, according to the different stages of the disease illustrated in Fig. 1b. Figure 4 summarizes our results. Quite interestingly, the force manifests a significant change when the disease progressively increases, showing characteristic resonances whose position and peak-topeak amplitude is strongly wavelength dependent. For each frequency, the optical forces manifests a monotonic decrease from the healthy stage (hRBG) to the diseased case (sRBC). This change is quite dramatic at the specific wavelengths where the hemoglobin is strongly absorbing, showing that the optical properties of the molecule are the dominant factor in the determination of the force. At the wavelength of $425 \mathrm{~nm}$, the optical force manifest an impressive change from approximately $120 \mathrm{pN}$ for $\mathrm{hRBC}$ to $40 \mathrm{pN}$ in the case of sRBC, with a reduction of $300 \%$.

These results can be relevant in the design and realization of new types of microfluidic channels [39-42] for the diagnosis and treatment of blood disease, such as malaria, where healthy blood cells are all optically selected and diseased cells are filtered out, opening new perspectives in optical nanomedicine.

\section{Conclusions}

In this work we presented an ab-initio analysis on the optical forces acting on biological objects. As an interesting case study, we considered red blood cells subjected to malaria disease. Optical forces are calculated from the Maxwell stress tensor formalism under a massively parallel FDTD campaign. Our results show that, even in the absence of any focused beam (as those used in trapping or tweezing experiments) the force is highly sensitive to changes in the cell morphology and sufficiently high (in the hundreds of $\mathrm{pN}$ ) range to sort the particles according to their ill conditions. This opens to the development of new techniques for the in-vivo treatment of diseases that affect the morphology of biological matter. 


\section{References}

[1] L. Novotny, B. Hecht, Principles of nano-optics, 2nd ed., Cambridge University Press, Cambridge, 2012.

[2] J. Chen, J. Ng, Z.F. Lin, C.T. Chan, Optical pulling force, Nat Photonics, 5 (2011) 531-534.

[3] A. Ashkin, Acceleration and Trapping of Particles by Radiation Pressure, Phys Rev Lett, 24 (1970) 156-\&.

[4] L. Boyde, K.J. Chalut, J. Guck, Interaction of Gaussian beam with near-spherical particle: an analytic-numerical approach for assessing scattering and stresses, J. Opt. Soc. Am. A, 26 (2009) 1814-1826.

[5] L. Boyde, A. Ekpenyong, G. Whyte, J. Guck, Elastic theory for the deformation of a solid or layered spheroid under axisymmetric loading, Acta Mech, 224 (2012) 819-839.

[6] L. Boyde, A. Ekpenyong, G. Whyte, J. Guck, Comparison of stresses on homogeneous spheroids in the optical stretcher computed with geometrical optics and generalized LorenzMie theory, Appl Optics, 51 (2012) 7934-7944.

[7] M. Capitanio, F.S. Pavone, Interrogating biology with force: single molecule high-resolution measurements with optical tweezers, Biophysical journal, 105 (2013) 1293-1303.

[8] K.C. Neuman, A. Nagy, Single-molecule force spectroscopy: optical tweezers, magnetic tweezers and atomic force microscopy, Nat Methods, 5 (2008) 491-505.

[9] D.B. Ruffner, D.G. Grier, Optical Conveyors: A Class of Active Tractor Beams, Phys Rev Lett, 109 (2012).

[10] O. Brzobohaty, V. Karasek, M. Siler, L. Chvatal, T. Cizmar, P. Zemanek, Experimental demonstration of optical transport, sorting and self-arrangement using a 'tractor beam', Nat Photonics, 7 (2013) 123-127.

[11] V. Kajorndejnukul, W.Q. Ding, S. Sukhov, C.W. Qiu, A. Dogariu, Linear momentum increase and negative optical forces at dielectric interface, Nat Photonics, 7 (2013) 787-790.

[12] M.P. MacDonald, S. Neale, L. Paterson, A. Richies, K. Dholakia, G.C. Spalding, Cell cytometry with a light touch: Sorting microscopic matter with an optical lattice, J Biol Reg Homeos Ag, 18 (2004) 200-205.

[13] M.C. Zhong, X.B. Wei, J.H. Zhou, Z.Q. Wang, Y.M. Li, Trapping red blood cells in living animals using optical tweezers, Nat Commun, 4 (2013).

[14] A. Taflove, S.C. Hagness, Computational electrodynamics : the finite-difference time-domain method, 3rd ed., Artech House, Boston, 2005.

[15] M.J. Piketmay, A. Taflove, J.B. Troy, Electrodynamics of Visible-Light Interactions with the Vertebrate Retinal Rod, Opt Lett, 18 (1993) 568-570.

[16] R. Drezek, A. Dunn, R. Richards-Kortum, A pulsed finite-difference time-domain (FDTD) method for calculating light scattering from biological cells over broad wavelength ranges, Opt Express, 6 (2000) 147-157.

[17] B. Simon, C.A. DiMarzio, Simulation of a theta line-scanning confocal microscope, J Biomed Opt, 12 (2007).

[18] J.D. Jackson, Classical electrodynamics, Wiley, New York,, 1962.

[19] J.Q. Lu, R.S. Brock, P. Yang, X.H. Hu, Modeling of light scattering by single red blood cells with the FDTD method, Nato Sci Ser Ii Math, 238 (2007) 213-242.

[20] J.P. He, A. Karlsson, J. Swartling, S. Andersson-Engels, Light scattering by multiple red blood cells, J Opt Soc Am A, 21 (2004) 1953-1961.

[21] L. Bi, P. Yang, Modeling of light scattering by biconcave and deformed red blood cells with the invariant imbedding T-matrix method, J Biomed Opt, 18 (2013) 055001-055001.

[22] X. Li, P.M. Vlahovska, G.E. Karniadakis, Continuum- and particle-based modeling of shapes and dynamics of red blood cells in health and disease, Soft matter, 9 (2013) 28-37. 
[23] M. Hammer, D. Schweitzer, B. Michel, E. Thamm, A. Kolb, Single scattering by red blood cells, Appl Optics, 37 (1998) 7410-7418.

[24] Y. Kim, J.M. Higgins, R.R. Dasari, S. Suresh, Y. Park, Anisotropic light scattering of individual sickle red blood cells, J Biomed Opt, 17 (2012).

[25] E. Evans, Y.C. Fung, Improved measurements of the erythrocyte geometry, Microvascular research, 4 (1972) 335-347.

[26] M. Dao, C.T. Lim, S. Suresh, Mechanics of the human red blood cell deformed by optical tweezers, Journal of the Mechanics and Physics of Solids, 51 (2003) 2259-2280.

[27] Y. Park, M. Diez-Silva, G. Popescu, G. Lykotrafitis, W. Choi, M.S. Feld, S. Suresh, Refractive index maps and membrane dynamics of human red blood cells parasitized by Plasmodium falciparum, PNAS, 105 (2008) 13730-13735.

[28] Y.M. Serebrennikova, J. Patel, L.H. Garcia-Rubio, Interpretation of the ultraviolet-visible spectra of malaria parasite Plasmodium falciparum, Appl Optics, 49 (2010) 180-188.

[29] N. Garcia, E. Stoll, Monte-Carlo Calculation for Electromagnetic-Wave Scattering from Random Rough Surfaces, Phys Rev Lett, 52 (1984) 1798-1801.

[30] T. Ye, N. Phan-Thien, Boo C. Khoo, Chwee T. Lim, Stretching and Relaxation of MalariaInfected Red Blood Cells, Biophysical journal, 105 (2013) 1103-1109.

[31] A. Esposito, J.B. Choimet, J.N. Skepper, J.M.A. Mauritz, V.L. Lew, C.F. Kaminski, T. Tiffert, Quantitative Imaging of Human Red Blood Cells Infected with Plasmodium falciparum, Biophysical journal, 99 (2010) 953-960.

[32] Y.M. Serebrennikova, J. Patel, W.K. Milhous, L.H. Garcia-Rubio, Quantitative analysis of morphological alterations in Plasmodium falciparum infected red blood cells through theoretical interpretation of spectral measurements, J Theor Biol, 265 (2010) 493-500.

[33] M. Friebel, M. Meinke, Model function to calculate the refractive index of native hemoglobin in the wavelength range of 250-1100 nm dependent on concentration, Appl Optics, 45 (2006).

[34] A. Roggan, M. Friebel, K. Do Rschel, A. Hahn, G. Mu Ller, Optical Properties of Circulating Human Blood in the Wavelength Range 400-2500 nm, J Biomed Opt, 4 (1999) 36-46.

[35] M. Daimon, A. Masumura, Measurement of the refractive index of distilled water from the near-infrared region to the ultraviolet region, Appl Optics, 46 (2007) 3811-3820.

[36] M. Friebel, M. Meinke, Determination of the complex refractive index of highly concentrated hemoglobin solutions using transmittance and reflectance measurements, J Biomed Opt, 10 (2005).

[37] C. Liu, A. Di Falco, D. Molinari, Y. Khan, B.S. Ooi, T.F. Krauss, A. Fratalocchi, Enhanced energy storage in chaotic optical resonators, Nat Photonics, 7 (2013) 474-479.

[38] J. Voros, The density and refractive index of adsorbing protein layers, Biophysical journal, 87 (2004) 553-561.

[39] F. Bragheri, P. Minzioni, R.M. Vazquez, N. Bellini, P. Paiè, C. Mondello, R. Ramponi, I.

Cristiani, R. Osellame, Optofluidic integrated cell sorter fabricated by femtosecond lasers, Lab on a Chip, 12 (2012) 3779-3784.

[40] J.H. Jung, K.H. Lee, K.S. Lee, B.H. Ha, Y.S. Oh, H.J. Sung, Optical separation of droplets on a microfluidic platform, Microfluidics and Nanofluidics, 16 (2014) 635-644.

[41] C. Faigle, F. Lautenschläger, G. Whyte, P. Homewood, E. Martín-Badosa, J. Guck, A monolithic glass chip for active single-cell sorting based on mechanical phenotyping, Lab on a Chip, 15 (2015) 1267-1275.

[42] M.M. Wang, E. Tu, D.E. Raymond, J.M. Yang, H. Zhang, N. Hagen, B. Dees, E.M. Mercer, A.H. Forster, I. Kariv, P.J. Marchand, W.F. Butler, Microfluidic sorting of mammalian cells by optical force switching, Nat Biotech, 23 (2005) 83-87. 


\section{Figures}

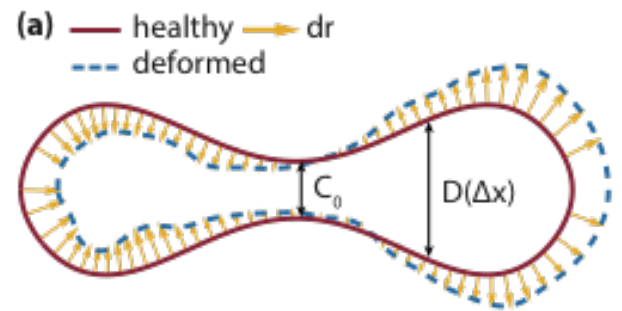

(b)

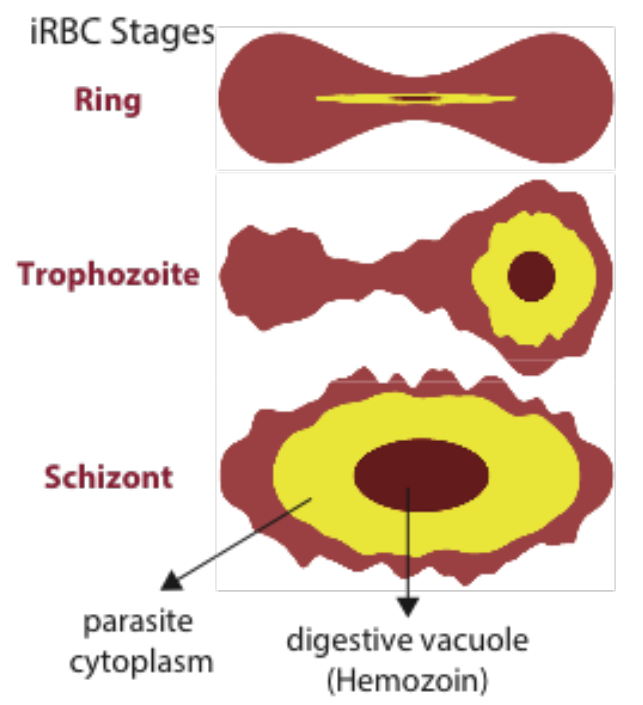

Figure 1. Analytic representation of healthy and P. Falciparum infected RBC. (a) In the Evans-Fung model, the profile of a healthy RBC (solid red line) can be represented in terms of the cell thickness $\mathrm{D}(\mathrm{x})$ (Eq. 1). In the presence of an infection, conversely, the profile of the cell undergoes complex morphological changes and the deformed cell (blue dashed line) can be modeled in terms of a deformation factor dr (yellow arrows) which takes into account both asymmetry and membrane fluctuations (Eqs. 2 and 3). (b) By combining Eqs. 1-3, we can model the three phenotypes of iRBC occurring in the presence of a P. Falciparum infection. In addition to the variation of the cell profile, we included the structural changes occurring in the interior of the RBC due to the growth of the parasite. 
(a)
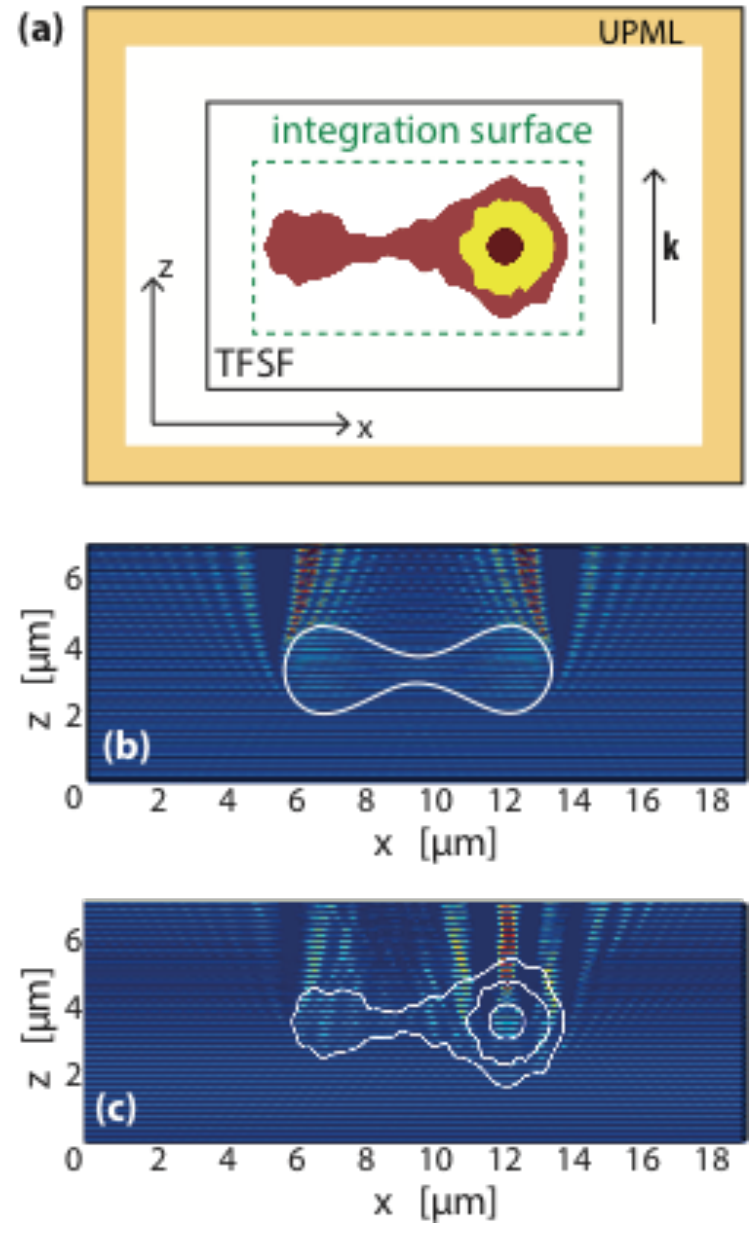

Figure 2. FDTD Setup. (a) FDTD setup: we enclosed the RBC within a rectangular TFSF region (solid black line). The force $\mathrm{F}_{\mathrm{z}}$ is measured along the propagation direction by integrating the Maxwell Stress Tensor (Eq. 4) over a surface (green dashed line) enclosing the cell. (b-c) Spatial electromagnetic energy distribution at the wavelength $\lambda=460 \mathrm{~nm}$ in the case of (b) a healthy cell and (c) a trophozoite iRBC. As can be evinced from the figure, in the later stages of the infection the digestive vacuoles of the parasite, rich in Hemozoin, produce a completely different scattering pattern. 

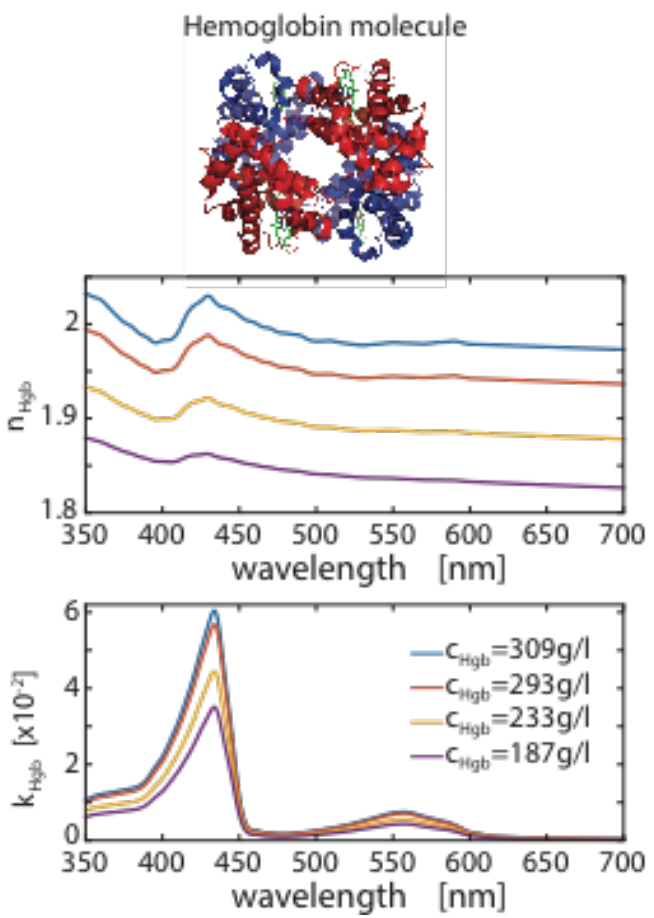

Figure 3. Optical properties of iRBC cytoplasm. (a) Real and (b) imaginary parts of the Hemoglobin refractive index as a function of wavelength and Hgb Concentration. Across the different stages of infection, the dispersion and absorption properties of the RBC are linked to the decrease of $\mathrm{Hgb}$ concentration in the cytoplasm. In our simulations we considered complex a refractive index corresponding to Hgb concentrations of 309g/l (healthy cell), 293g/l (rRBC), $233 \mathrm{~g} / \mathrm{l}(\mathrm{tRBC})$ and $187 \mathrm{~g} / \mathrm{l}$ (sRBC) [27]. 


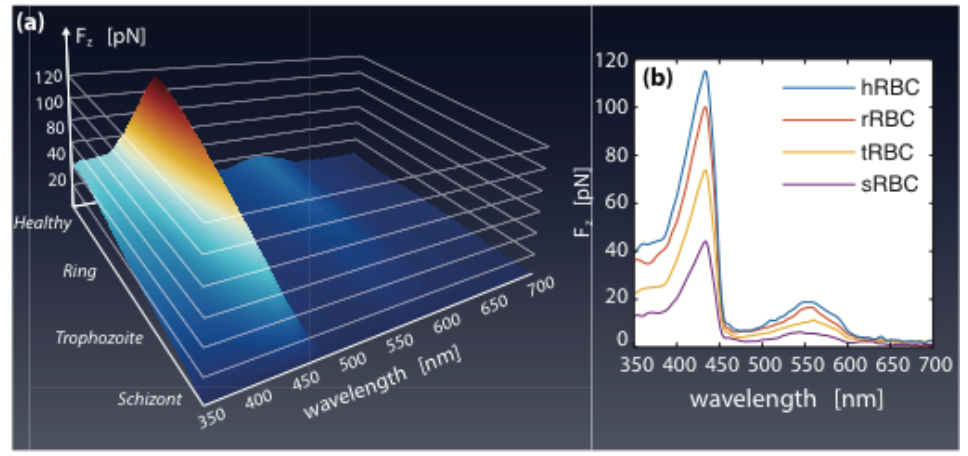

Figure 4. Optical forces on iRBC at different stages of P. Falciparum infection: FDTD Results. (a) Surface plot of the optical force $F_{z}$ as a function of wavelength and disease progression. Due to the strong decrease in Hgb concentration, the optical force exhibits up to a $300 \%$ variation between a healthy RBC (b, blue solid line) and an iRBC in an advanced infection stage (b, violet line). 